\title{
Fp1 Prosthesis in Maxillary Ridge Defect and Fixed Partial Denture in Mandibular Ridge Defect - A Case Report
}

\author{
Ashish. R. Jain* \\ Department of Prosthodontics, Tagore Dental College and Hospitals, Chennai, India \\ *Corresponding author: dr.ashishjain_r@yahoo.com
}

Received November 12, 2014; Revised December 07, 2014; Accepted December 11, 2014

\begin{abstract}
Severely resorbed alveolar ridge in aesthetic zone of maxilla and mandible can compromise the successful implant placement and aesthetic outcome. Advancements in the field of dental implant therapy have lead to predictable survival rates of dental implants. The current definition of success in addition to long-term predictability, function and integration of the implant focuses on esthetic considerations. This article presents a case report of severely deficient maxillary and mandibular alveolar ridge where implant placement in the Maxillary aesthetic zone has been done in a staged approach as there was inadequate supporting bone and soft tissue and a conventional fixed partial denture in mandibular anterior region.
\end{abstract}

Keywords: conventional FPD, implant retained fixed Fp-1 prosthesis, Bone Grafting

Cite This Article: Ashish. R. Jain, "Fp1 Prosthesis in Maxillary Ridge Defect and Fixed Partial Denture in Mandibular Ridge Defect - A Case Report.” International Journal of Dental Sciences and Research, vol. 2, no. 6 (2014): 184-189. doi: 10.12691/ijdsr-2-6-16.

\section{Introduction}

Advancements in the field of dental implant therapy have lead to predictable survival rates of dental implants. [1] The current definition of success in addition to longterm predictability, function and integration of the implant focuses on esthetic considerations. [2] In the anterior maxilla this is more critical due to the visibility of the region and if a high lip line is present, the smile line is more revealing thus increasing the need for an esthetic result, with some authors ranking function and aesthetics in the anterior maxillary region to be of equal importance [3].

The esthetic component of an implant is dictated by the soft tissue framing, which is defined as the gingival contour that surrounds the prosthesis, this includes the preservation of the interdental papillae and a gingival margin which is symmetrical with the adjacent gingival architecture. [4] The interdental papilla to completely fill the space between teeth or implants can be achieved by having the crest of the interproximal bone within $5 \mathrm{~mm}$ of the proposed contact point of the final restoration. [5] An ideal emergence profile is achieved when there is proper placement of the implant in a correct three dimensional position. [6] The centering of the implant mesiodistally is important and requires a minimum of $1.5 \mathrm{~mm}$ of space between the implant and the adjacent natural tooth or implant, if this distance is violated, than a loss of interdental papilla is expected.
Correction of bone defects can be done in a variety of ways which include barrier membranes with guided bone regeneration, onlay grafts, block grafts or distraction osteogenesis [7].

Guided bone regeneration is a predictable method for the regeneration of adequate volume of bone for implant placement and can be done simultaneously with implant placement or in a staged approach. [8] Even though immediate implant placement has shown comparable success rates a delayed or staged placement may be a better choice in esthetic areas when there are defects observed [9]. This article illustrates a case report of severely deficient maxillary and mandibular alveolar ridge where implant placement in the Maxillary aesthetic zone has been done in a staged approach as there was inadequate supporting bone and soft tissue and a conventional fixed partial denture in mandibular anterior region.

\section{Case Description}

A 29-year-old male patient reported, complaining of inability to chew, unesthetic appearance of face, difficulty in speech and desired replacement of missing teeth in upper and lower front tooth region of jaw. His medical history revealed a history of depression and anxiety that was related to his marital status. His dental history revealed a fall on his face that he said occurred at least ten years ago, and lost his following teeth $(12,11,21,22,23.33,32,31)$. A detailed examination revealed oral hygiene was satisfactory and his periodontal 
pocket depths were under $3 \mathrm{~mm}$ in all remaining teeth. A full mouth radiograph and CT scan was obtained (Figure 1). CT scan revealed that there is only $1.7 \mathrm{~mm}$ bone width in maxillary anterior arch and $1.1 \mathrm{~mm}$ in mandibular anterior arch also adequate ridge height at the implant site was insufficient and necessitate for horizontal bone augmentation. Radiographically the clinical findings were verified and additionally revealed vertical bone loss and horizontal bone loss that was limited to the region of
$(12,11,21,22,23.33,32,31,41)$. The patient was presented with different treatment options, after discussing the pros and cons of each and the following treatment option was agreed upon; with concurrent bone and tissue grafting, followed by implant placement in maxillary anterior region and Conventional fixed partial denture in mandibular arch. During the healing phase a transitional removable partial denture would be fabricated for esthetics and function.

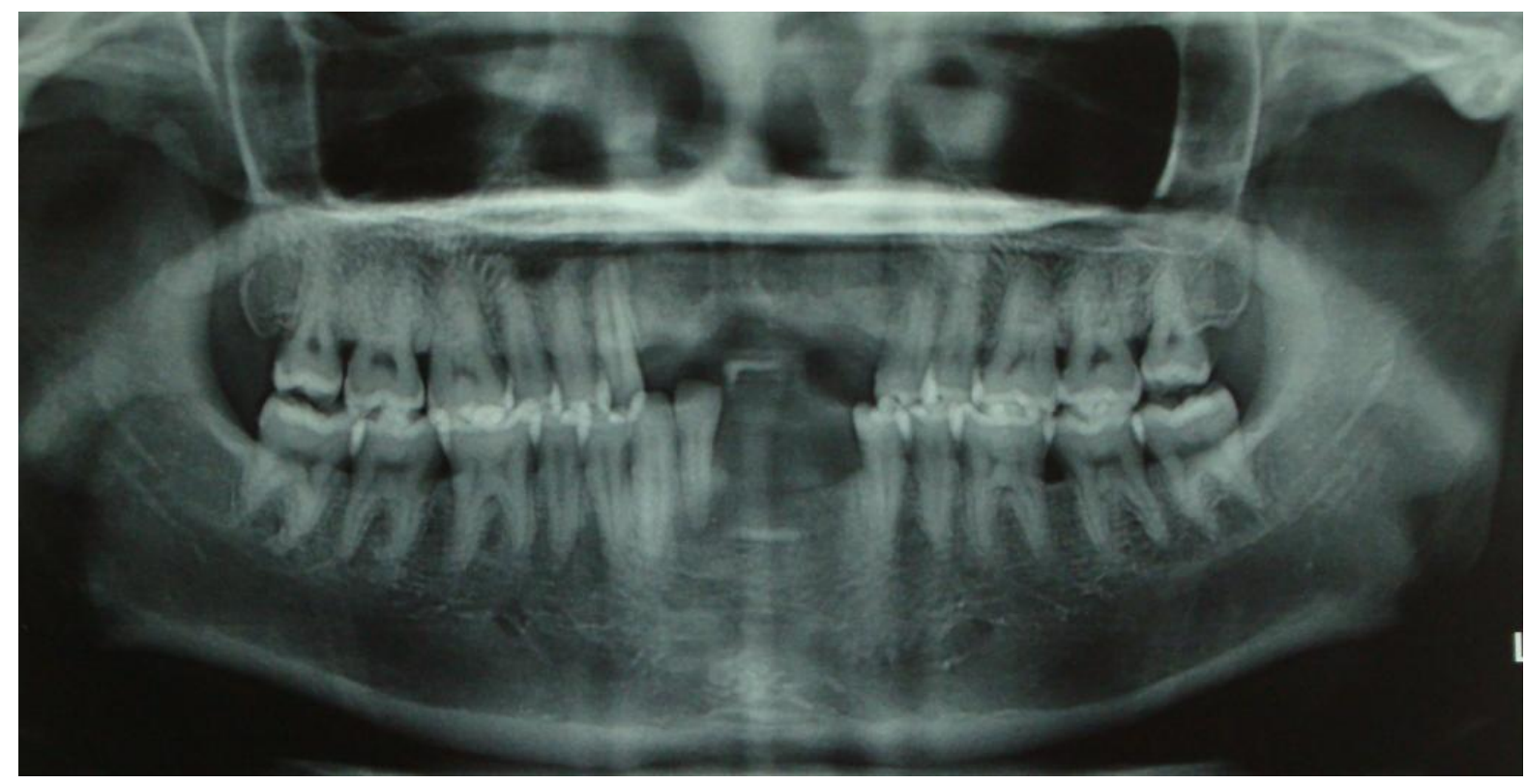

Figure 1. Radiograph after 4 months of Graft placement

\section{Procedure}

Alginate impressions were made of both arches and poured in Type III dental stone. Shade was selected and the casts were hand-articulated in maximum intercuspation (MI) and sent to the lab for the fabrication of a transitional maxillary partial denture.

\subsection{Grafting Procedures}

Donor Site: The surgical procedure was performed under local anesthesia (2\% lidocaine containing 1:1,00,000 epinephrine). A full thickness incision is given distal to the most posterior tooth and continues posteriorly to the retro molar pad and ascending ramus and anteriorly in the buccal sulcus opposite to the first premolar, where an oblique releasing incision is given to the depth of the vestibule. A full thickness mucoperiosteal flap is reflected to permit visualization of the external oblique ridge, buccal shelf, lateral ramus, and mental neurovascular bundle. The ramus donor site is lateral to the molar (buccal shelf region) and extending up to the ascending ramus. A superior osteotomy is created approximately 4-5 mmmedial to the external oblique ridge with the help of $702 \mathrm{~L}$ straight fissure bur. It begins opposite to the distal half of the mandibular first molar or opposite to the second molar and continues posteriorly in the ascending ramus. The length of this osteotomy depends on the graft size. The vertical osteotomies begin at each end of the superior bone cut and continue inferiorly approximately 10-12 mm. All osteotomies just penetrate only through buccal cortex into marrow. Finally, a $0.8 \mathrm{~mm}$ round bur is used to create a groove that connects the inferior aspect of each vertical osteotomy. The graft is then harvested using bone spreader or chisel that is malleted along the superior osteotomy. The block graft is stored in normal saline.

Recepient Site: A mid-crestal incision with bilateral oblique releasing incision is given in maxillary anterior region on the implant site and full thickness mucoperiosteal flap is elevated. The recipient site is perforated with $0.8 \mathrm{~mm}$ round bur to penetrate underlying marrow. This preparation provides access for trabecular bone blood vessels to the graft and accelerates revascularization. Surgical trauma also accelerates regional acceleratory phenomenon which results 2-10 times faster healing. Post-surgically amoxicillin 500mg TID for five days and Chlorhexidine $0.12 \% 30 \mathrm{ml}$ rinse BID for seven days was prescribed, while Ibuprofen 200mg was recommended for pain relief. The patient was put on a two week, one month and three month recall ensuring the proper management of the edentulous site.

\subsection{Stage I implant Surgery}

Six months after the grafting procedure a radiograph of the edentulous site was taken which revealed adequate bone volume, bone sounding was done and it was felt that there was about $7 \mathrm{~mm}$ of bone in a bucco-palatal direction. It was decided that implant placement could be attempted at this time.. A full thickness mucoperiosteal flap was raised in the maxillary arch from left first premolar region to right Canine region. Three implants $(3.75 \times 13 \mathrm{~mm})$ (Self thread, Touareg ${ }^{\mathrm{TM}}$ NP, Adin dental implant system, Israel) were placed and primary stability was achieved at 
$35 \mathrm{~N}$, cover screw was placed on top of the implant The implant dimensions were selected with the help of the CT scan. A total of three implants were placed in the maxillary anterior arch .The flap was closed using horizontal interrupted sutures. After 1 week, the sutures were removed and an transitional denture was modified and inserted. A post surgical radiograph was taken, and it showed adequate placement (Figure 2).

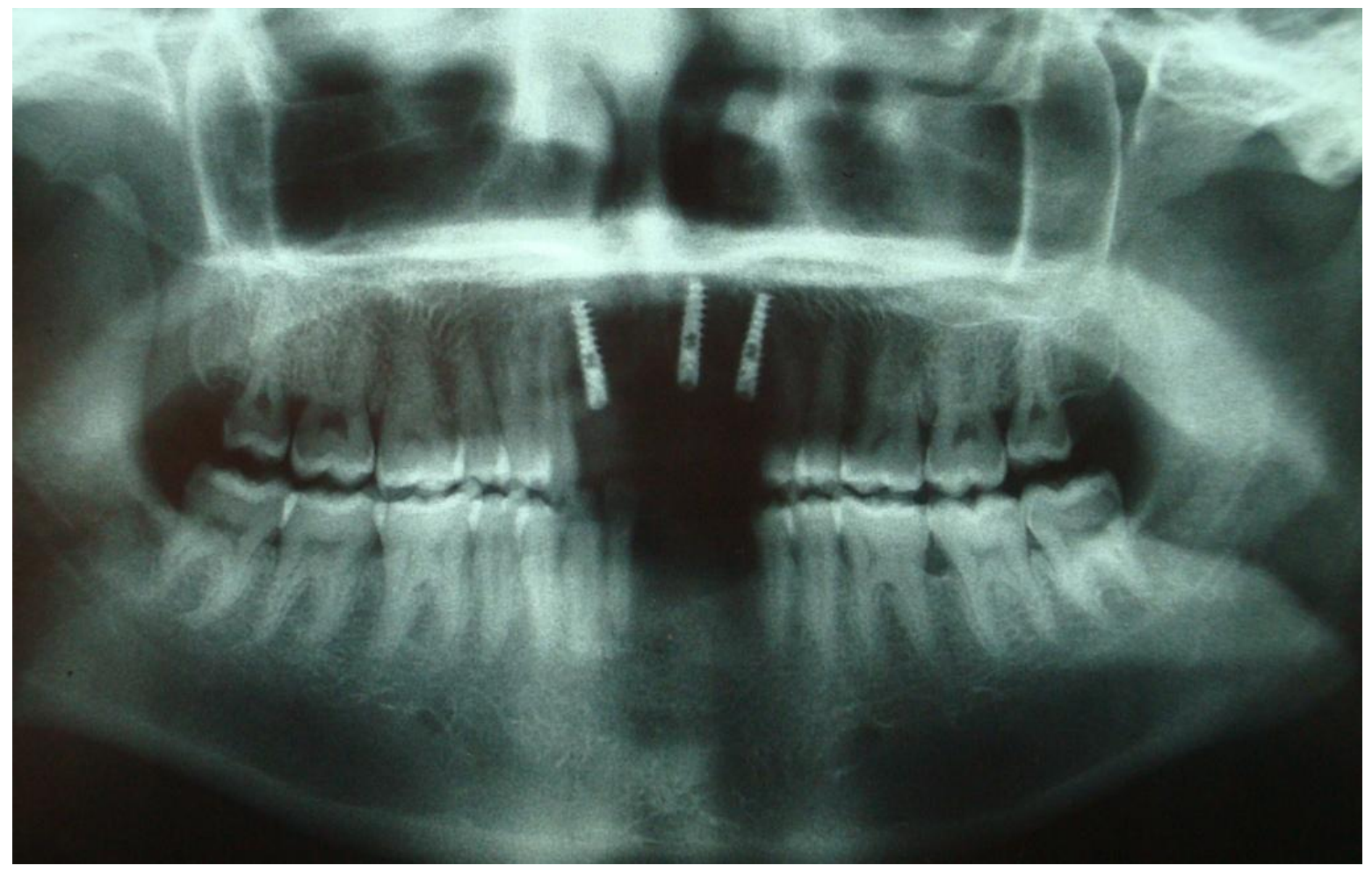

Figure 2. Radiograph after six months of implant placement

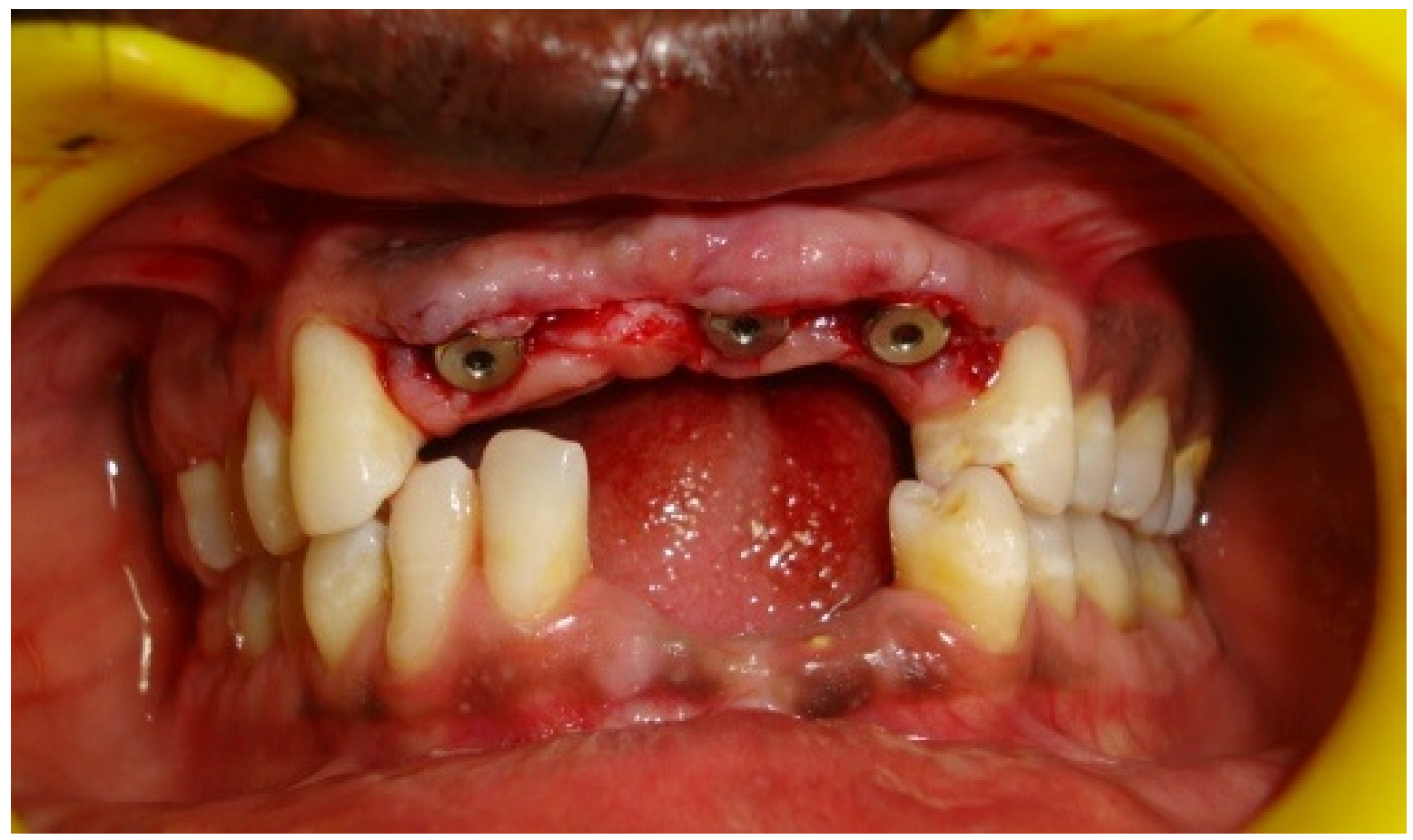

Figure 3. Cover screw exposed and Healing abutments in place

\subsection{Stage II Implant Surgery}

After a waiting period of 4 months an OPG was obtained to evaluate the bone to implant contact percentage and later stage II surgery was performed under local anaesthesis. Cover screws were exposed and healing abutments were placed and the flap sutured (Figure 3).

\subsection{Prosthetic Phase}

Maxillary and mandibular arch impressions were made using alginate (Tulip Alginate Impression Material, Cavex, Holland Bv, Haarlem Holland). (Figure 4a, Figure 4b). A custom open tray was fabricated in acrylic resin (Autopolymerizing acrylic resin, ALIKE ${ }^{\mathrm{TM}}$; GC America, 
ALSIP, USA) for the maxillary arch. The open tray was verified in the patient's mouth. One week later, healing caps were removed from the maxillary anterior implants and impression copings were attached to them. These open tray impressions copings were stabilized with 23 guage ortho wire and blocked out with acrylic resin (Autopolymerizing acrylic resin, ALIKETM; GC America, ALSIP, USA) (Figure 5). The maxillary arch impression was made with putty and monophase polyvinyl siloxane impression material (Aquasil Lv Ultra, Smart Wetting Impression Material, Dentsply, Detrey Gmbh, Konstanz, Germany) (Figure 6a).Tooth preparation was completed in mandibular acrh for conventional fixed partial denture from right canine to left first premolar and impression was made using putty and addition silicone impression material (Aquasil Lv Ultra, Smart Wetting Impression Material, Dentsply, Detrey Gmbh, Konstanz, Germany). (Figure 6b).
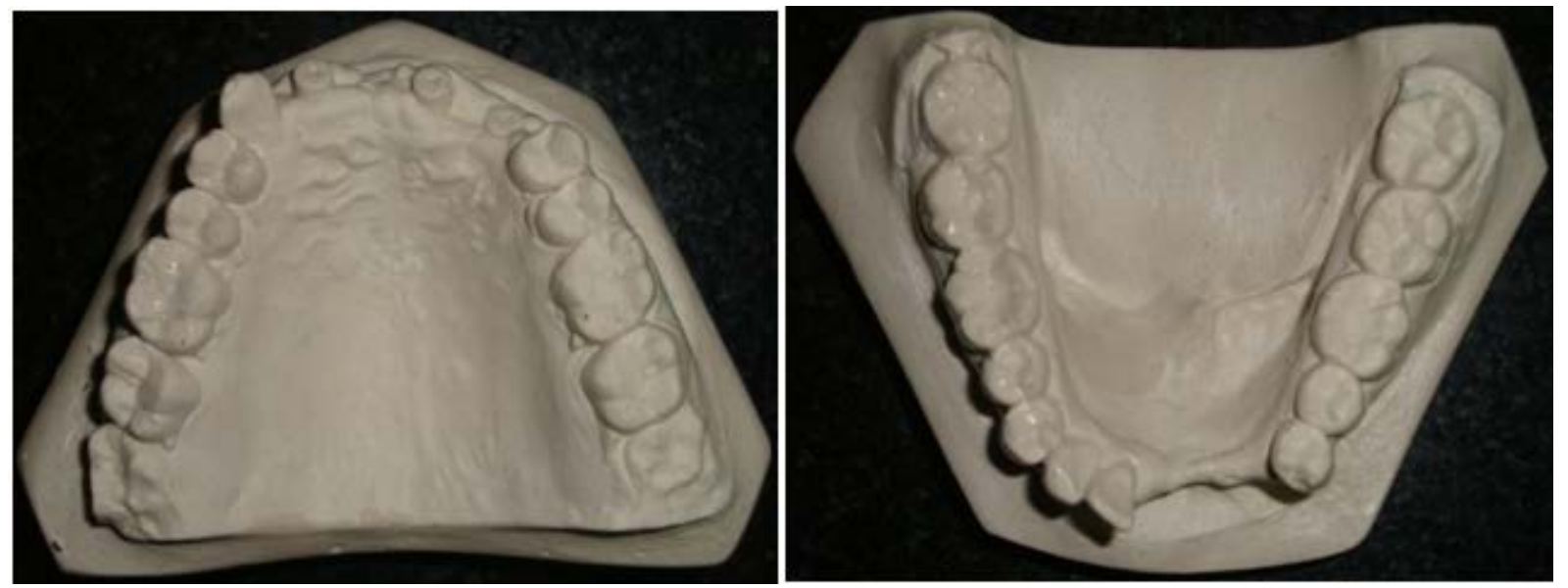

Figure 4. Maxillary and Mandibular primary casts

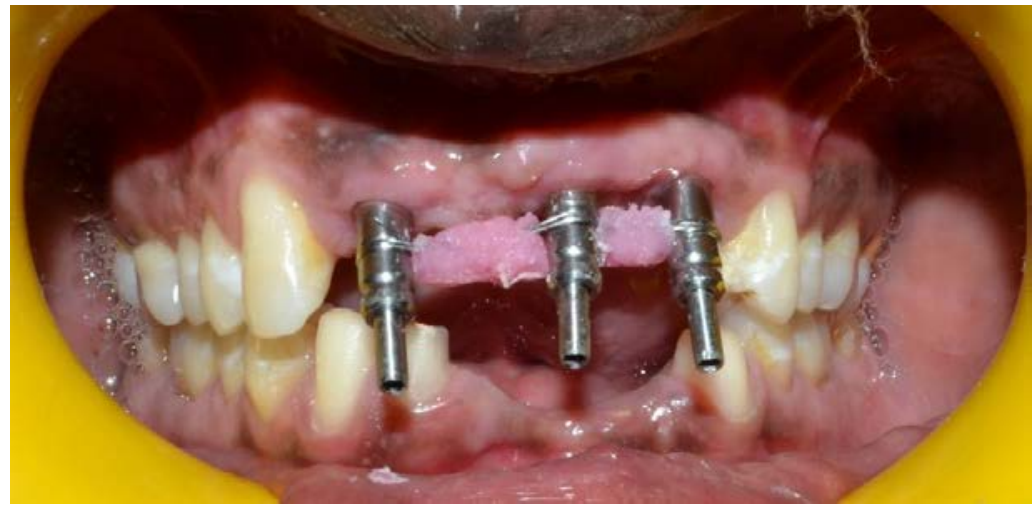

Figure 5. Adjustment and Verification of open tray in patient mouth. Stabilization of impression copings in patient mouth
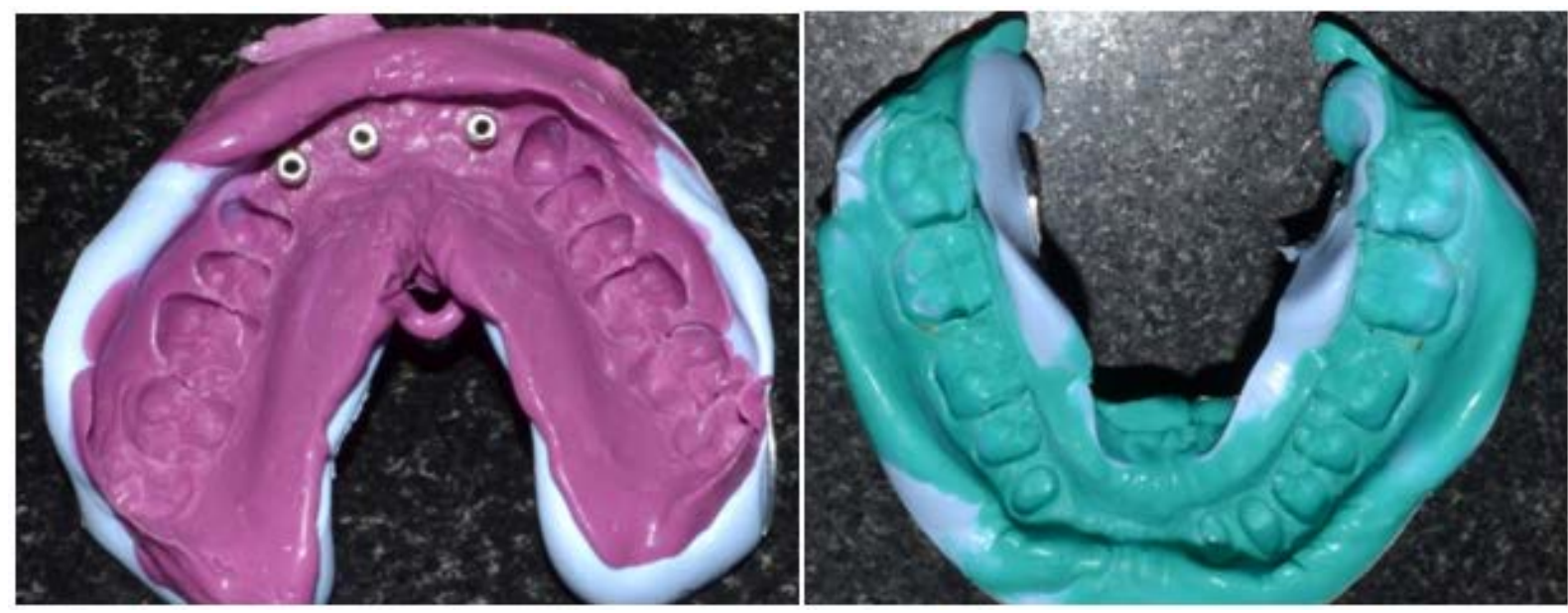

Figure 6. Maxillary And Mandibular Final impressions

The casts were poured in die stone (Ultrarock, Kalabhai Karson Pvt Ltd, Mumbai, India) .The abutments were evaluated in the cast and patients mouth for parallelism (Figure 7a, Figure 7b). It was found that two of the three abutments required an angulation of 15 degree for parallelism. Abutments were tried and prepared in the patients mouth (Figure 8). The abutments were further milled in the lab to attain parallelism and a shoulder finish line. A mock wax up was done using which a metal framework was fabricated for the Porcelain fused to metal 
prosthesis. A four unit PFM implant prosthesis was fabricated in the maxillary arch and six unit PFM fixed partial denture prosthesis was fabricated in the mandibular arch. The prosthesis was cemented (Fuji, Type I, GC, Japan) on the abutments after evaluation. Occlusion was verified with an articulating paper (Ardent Horseshoe style, Whipmix
Corp., Louisville, Kentucky, USA) which had a thickness of $63 \mu \mathrm{m}$ to ensure balance during lateral movements. The patient was pleased with the esthetic and functional outcome of the Prosthesis (Figure 9a, Figure 9b, Figure 9c). The patient was advised on oral hygiene maintenance. The patient was recalled for review every 6 months.

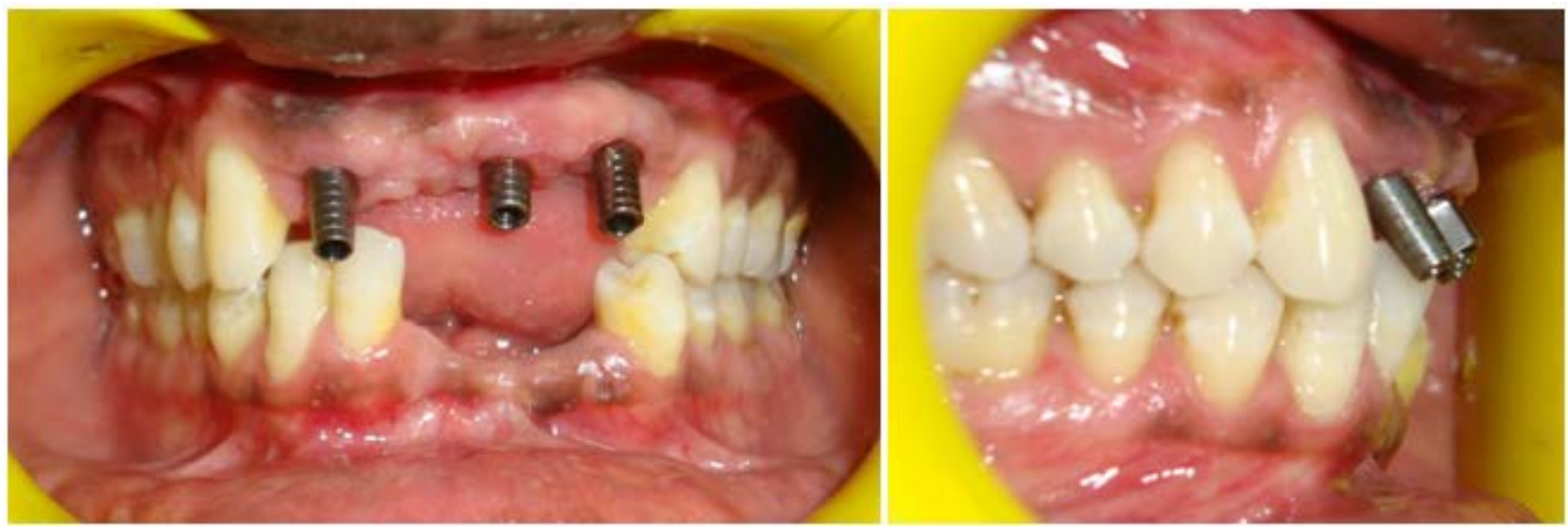

Figure 7. a) Verification of abutments for parallelism. b) Abutment placement and modification intraorally

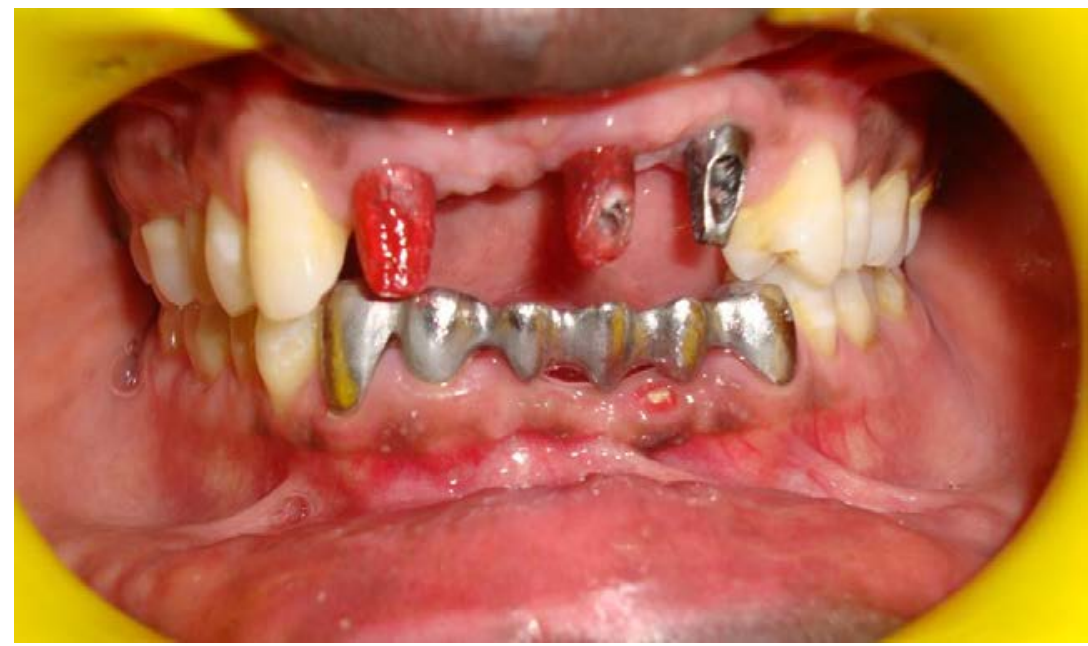

Figure 8. Metal try in intraorally
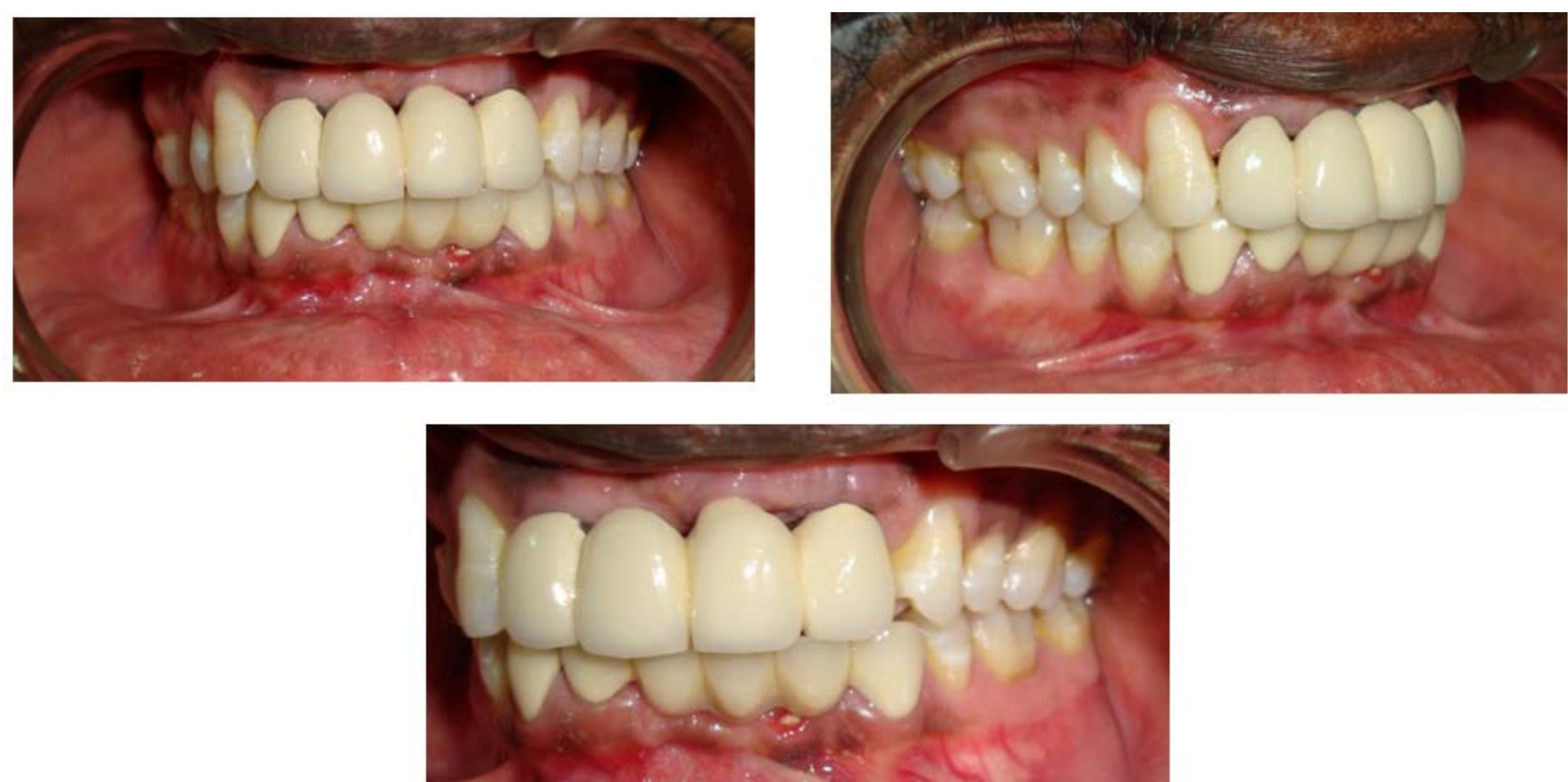

Figure 9. Maxillary and Mandibular prosthesis in place 


\section{Discussion}

Implants have become an integral part of prosthodontic rehabilitation. Their versatility allows their use in both removable and fixed prostheses. When placement of sufficient number of implants is feasible, the superstructure can be totally implant retained. Careful intraoral examination is crucial to estimate the available inter-arch distance and fabricate the final prosthesis with the necessary strength, esthetics, and cleansability [1-6].

Implant retained fixed prostheses have been shown to improve the quality of life for patients and contribute to the well-being of the patient's psychology. It was planned in the maxillary arch because of esthetics as prime concern (visibility, lip line), where as conventional fixed partial denture for mandibular arch because lack of sufficient bone in width and as visibility was not a problem.soft tissue defects were covered with pink porcelain. The benefits of a pink porcelain design for the cervical portion of a definitive prosthesis have been discussed by Potashnick. Such a design was considered for this patient to compensate for the severe soft-tissue and bone loss experienced following tooth removal. Occlusal forces have been found to have increased considerably following the placement of an implant retained prosthesis. Residual ridge width of an edentulous maxilla decreases gradually in incisor and canine areas. However, the decrease in maxillary residual ridge width is small and not associated with the type of mandibular restoration [9].

\subsection{Clinical Relevance to Interdisciplinary Dentistry}

To help the restorative dentist to identify the ideal implant receptor site.

Sound periodontal and restorative status is prerequisite to consider implant treatment as an option.

Restorative driven implant placement is paramount for overall integrity of the dentition.

\section{Conclusion}

Every patient has unique treatment needs. Proper diagnosis and treatment plan are important but cannot be all inclusive. A comprehensive examination, including a thorough medical and dental history, orofacial and dental clinical examination, dental radiographs, impressions, and jaw relation records for mounting casts are important steps leading to a successful oral rehabilitation. Careful integration and sequencing of the different areas of treatment needed, enhances the final result. Dentists must consider the advantages and disadvantages the available implant prosthetic options and match them to the patient's expectations. This article reports implant retained fixed prosthesis in the Maxillary aesthetic zone has been done in a staged approach as there was inadequate supporting bone and soft tissue and a conventional fixed partial denture in mandibular anterior region. Occlusion and articulation were found to be good over a period of two years.

\section{References}

[1] Henry PJ. A review of guidelines for implant rehabilitation of the edentulous maxilla. J Prosthet Dent 2002; 87: 281-8.

[2] Kelly E. Changes caused by a mandibular removable partial denture opposing a maxillary complete denture. J Prosthet Dent 1972; 27: 140-50.

[3] Barber HD, Scott RF, Maxson BB, Fonseca RJ. Evaluation of anterior maxillary alveolar ridge resorption when opposed by the transmandibularimplant. J Oral Maxillofac Surg 1990; 48: 1283-7.

[4] Lechner SK, Mammen A. Combination syndrome in relation to osseointegratedimplant-supported overdentures: a survey. Int J Prosthodont 1996; 9: 58-64.

[5] Nevalainen MJ, Rantanen T, Närhi T, Ainamo A. Complete dentures inthe prosthetic rehabilitation of the elderly persons: five different criteria to evaluate the need for replacement. J Oral Rehabil 1997; 24: 251-8.

[6] Kleis WK, Kämmerer PW, Hartmann S, Al-Nawas B, Wagner W. A comparison of three different attachment systems for mandibular two-implant overdentures: One-year report. Clin Implant Dent Relat Res 2010; 12: 209-18.

[7] Mericske-Stern R. Treatment outcomes with implant-supported overdentures: Clinical considerations. J Prosthet Dent 1998;79:6673.

[8] Kent G, Johns R. Effects of osseointegrated implants on psychological and social well-being: a comparison with replacement removable prostheses. Int J Oral Maxillofac Implants 1994; 9: 103-6.

[9] Stafford D, Glantz PO, Lindqvist L, Strandman E. Influence of treatment with osseointegrated mandibular bridges on the clinical deformation of maxillary complete dentures. Swed Dent J Suppl 1985; 28: 117-35. 